\title{
Crucial Role of Diabetes Mellitus in Delayed Angiogenesis of Wound
}

\author{
Alok Raghav ${ }^{1 *}$ and Jamal Ahmad ${ }^{2}$ \\ ${ }^{1}$ Rajiv Gandhi Centre for Diabetes \& Endocrinology, J.N Medical College, Aligarh Muslim University Aligarh, India \\ ${ }^{2}$ Former Professor of Endocrinology, Rajiv Gandhi Centre for Diabetes \& Endocrinology, JN Medical College, Aligarh Muslim University, India
}

Submission: March 22, 2018; Published: April 10, 2018

*Corresponding author: Alok Raghav, PhD, Rajiv Gandhi Centre for Diabetes \& Endocrinology, JN Medical College, Aligarh Muslim University, India, Tel: +919412672185; Email: alokalig@gmail.com

Abstract

Diabetes mellitus is a chronic metabolic disorder that is characterized by uncontrolled hyperglycemia. Diabetes mellitus is associated with micro-and macro- vascular complications including delayed wound healing, chronic ulceration and lower extremity amputation that impaired the quality of life. In healthy state, the wound healing is defined and occurs orderly, but during uncontrolled hyperglycemia, it gets impaired at all stages of healing with poor vasculature growth and function. The delayed angiogenesis is a matter of serious concern as raised in this work.

Abbreviations: RAGE: Receptors Of Advanced Glycation End; BMI: Body Mass Index; DFU: Diabetic Foot Ulcers; PAD: Peripheral Arterial Disease; FGFs: Fibroblast Growth Factors; ECs: Endothelial cells; DR: Diabetic Retinopathy; DN: Diabetic Nephropathy; PEDF: Pigment Epithelium Derived Factor

\section{Introduction}

Diabetes mellitus (DM) is hub of metabolic disorders which are characterized by hyperglycemia (increased blood sugar) due to defective machinery of insulin secretion; insulin action may be contributed by both. Chronic and prolonged hyperglycemia in diabetes is associated with short term and long-term damage, organ failure, dysfunction, micro-and macro-vascular complications dealt with eyes, kidneys, nerves, heart and blood vessels [1]. Numerous pathogenesis mechanisms are involved in the production of diabetes. Among the most important and foremost mechanistic pathway is the destruction of insulin production $ß$-cells of the pancreas via autoimmune destruction highway that results in deficiency of insulin and give rises to hyperglycemia leading to DM. Insulin resistance also plays an important role in the development of DM due to insufficiency of insulin receptors to sense insulin. Prominent symptoms of hyperglycemia in DM are presented by polyuria, polydipsia, polyphagia, weight loss along with blurred vision. Chronic hyperglycemia invites a range of microbial infections along with the development of the life-threatening ketoacidosis or non-ketotic hypersomolar syndrome. Long-term complication associated with DM include potential loss or blurred vision (retinopathy); end-stage renal failure (nephropathy); peripheral neuropathy developing risk of diabetic foot ulcers, nontraumatic lower limb amputations and Charcot joints. Moreover, the autonomic neuropathy another complication causes the gastrointestinal, genitourinary, sexual dysfunction and cardiovascular complications. Macro-vascular complications associated with long-term damage include atherosclerosis.

In the present review, we will focus on the angiogenesis of diabetic wounds delayed due to chronic persistent hyperglycemia. The area of diabetic wound is still a topic of never ending debate, due to increasing prevalence of diabetes worldwide, the episodes of mortality and morbidity with impaired wound healing along with lower extremity amputations are showing increasing trends. In one of the recent study it was found that global prevalence of diabetic foot is 6.3\%, North America (13.0\%), Asia (5.5\%), Europe (5.1\%), Africa (7.2\%) and Oceania (3.0\%) [2]. It is also quoted by the previously published literature that diabetic foot wounds are more prevalent in males compared to females and also higher in type 2 diabetic patients compared to type 1 diabetic patients [2]. It is interesting to quote that diabetic foot ulcers are prevalent in older age patients with lower body mass index (BMI) having longer chronic duration of diabetes mellitus with more prevalent history of hypertension, diabetic retinopathy and smoking history [2]. Development of distal chronic ulcers in diabetes mellitus is also among the major cause of morbidity and mortality. 
Moreover, wound healing mechanism is an orchestrated activity, a plethora of factors should be noticed for chronic wounds initiation in diabetes mellitus, impaired angiogenesis, altered growth factors expression, disturbed receptors of advanced glycation end products (RAGE) signaling, elevated expression of inflammatory response at ulcer margins along with reduced cellular proliferation and centripetal cell migration. It is estimated that chronic wounds in diabetes mellitus costs around 12 billion US dollars expenditure in its healthcare related system dealt with its treatment and management [3]. Recently it is shown that endovascular treatment and vascular surgery of diabetic foot patients in New Zealand was found to be 30,000 New Zealand Dollar [4]. The statistical figure also demonstrated that more than 2 million wound's patients were treated in 2012 and 2013 in UK [5]. Therefore, most of the foot ulcers and chronic wounds are the prime cause of lower extremity amputations if left untreated.

\section{Classification of Wounds}

Wounds on the basis of underlying cause of wound makeup and creation boundaries are grouped into open and closed wounds and on the basis of wound healing physiology, further classified into acute and chronic wounds.

\section{Open Wound}

Blood exsanguinations continuously operate from open wounds with clearly visible bleeding. These wounds based on the object causing wounds further classified into various types [6]

\section{Incised Wound}

In this wound type, there is no loss of tissue with minimal damage at tissue level. It is created by a sharp and precise object or also may be due to slashing into skin. Profuse bleeding occurs, and immediate action should be taken into consideration to cure. These wounds are usually longer in length than deeper. Mostly, times these types of wounds are not very deep and only cause damage to skin. Occasionally, if these type of wounds are very deep, leading to cutting of muscle tissue, tendons or also to the blood vessels and capillaries causing life-threatening issues.

\section{Laceration Wounds or Tears Wounds}

This type of wound is generally occurs due to nonsurgical injury in relation to few traumatic conditions that results in tissue loss and damage. A laceration wound is also termed as tear wounds as it happened due to torn or cut open of skin, muscles or tissues. These wounds may be deep and shallow, short or long, narrow or wide. These are the resultant of skin hitting with some object with force. Laceration repair includes the procedure of cleaning, preparing and closing of wound. However minor laceration does not require medical attention.

\section{Abrasions or Superficial Wounds}

These types of wounds arises due to sliding or fall on any rough surface that causes superficial damage to skin and only epidermis portion received injury. Its severity is less than the laceration with minimal bleeding visibility. Mild abrasions do not forms scars rather than deep abrasions that may form scar tissue. Its severe form is also termed as avulsion that arises due to worst traumatic situations leading to removal of all layers of skin. These wounds are painful due to exposure of nerves at the wound site. On the basis of degree of abrasions wounds these are further grouped into three main types as:

a) First degree abrasion wound injury that involve epidermis

b) Second degree abrasion wound injury that involves epidermis and dermis.

c) Third degree abrasion wound injury that involved subcutaneous layers of skin.

\section{Puncture Wounds}

As the name itself telling that, these types of wounds are caused due to puncture of the skin with some pointed objects like needle or nail. These wound are deeper and may cause more bleeding. These wounds are also susceptible for bacterial infections because of dirt penetration to a deeper extent.

\section{Penetration Wounds}

These wounds are cause by the sharp objects that pierces the skin and damage the tissue of the body, thereby generating an open spaces or wound. In this episode, the penetrating objects pierce the tissue and come out of the body creating an opening e. in stabbing by knife.

\section{Gunshots Wounds}

A gunshot wound is a type of physical trauma created by some arms and munitions e.g by bullets. In this form, the bullet either stacked into the tissues or may exit after creating hole or damage to the tissue laving excessive bleeding.

\section{Closed wounds}

As the name implies itself, that the closed wounds are type of wounds, in which the bleeding initiate from any part of the body but remains inside leaving visible form of bruises. These wounds present below classifications and fatal as like as open wounds described above.

\section{Contusions and Bruises Wounds}

A contusion wound is commonly called as bruise wound. It is type of hematoma occurring in tissues creates damage to capillaries or venules by trauma thereby generating blood seep, hemorrhage.

\section{Hematomas or Blood Tumors}

It is also a form of wound in which the blood remains stagnant at particular place beneath the skin and causes internal damage. 


\section{Crush Injury}

Crush wound is formed as a resultant of extreme force for long time to the skin thereby damaging the body parts.

\section{Acute Wounds}

An acute wound is an injury form that occurs accidental rather than over time. These wounds can occur anywhere on the body and its degree vary from superficial to deeper thereby damaging nerves, blood vessels, skin, muscles etc. There are prominently two types of acute wounds are there;

a) Surgical Wounds: Incisions fulfilling some purpose of health care, created by some experts in managed way in sterile environment. These wounds may be closed after the purpose resolved.

b) Traumatic Wound: Injuries to skin by some extreme forces of some nature.

\section{Chronic Wounds}

Chronic wounds that failed to follow the mechanisms or stages of normal wound healing mechanism and therefore present pathological inflammation and complications [7]. These wound require prolonged time to heal up and their frequency of reoccurring is also fast. These wounds are caused by several factors but primarily include diabetes mellitus, local immunological reaction, infection, hypoxia, trauma, immunodeficiency etc. Some common example of chronic wound is diabetic foot ulcers (DFU).

\section{Diabetic Foot Ulcers}

The diabetic foot ulcers (DFU) are defined as the formation of non-healing or poorly healed wound, through the dermis especially in diabetes mellitus. The common sites for DFUs occurrence are plantar surface of foot (midfoot and metatarsal heads), toes (distal tip or dorsal interphalangeal joints). These are characterized by neuropathy that may be purely ischemic or neuroischemic (mixed) in association with peripheral arterial disease (PAD). DFUs are the leading cause of hospitalization of diabetic patients and counted as most serious and expensive complications associated with diabetes mellitus. In diabetic patients with peripheral neuropathy, repetitive stress over a wound area that is subject to high vertical shear stress is the prime cause of DFUs [8]. Peripheral arterial disease also contributes in the development of DFUs [8]. Moreover, the association of vasculature and the predisposition to infection makes a triad that ultimately leads to amputation.

\section{The Burden of Chronic Wounds}

Chronic wounds impart economic burden on society as revealed by earlier published studies [9-10]. In a recent study published by Jarbink et al. [11] have defined the property of chronic wounds that shows difficulty in healing as "one that has failed to proceed via an routinely and timely reparative mechanism to generate anatomic and functional integrity within a time span of 3 months or proceeded via repair process without undergoing sustained anatomic and functional outcome". Chronic wounds in the form of DFUs are considered to be silent killers and spreading as epidemic. The reason behind this epidemic is increasing rate of ageing populations that is severely associated with the reduce wound healing rates [12-13]. The episodes of chronic wounds has also exacerbated by global increase in obesity, diabetes mellitus and also cardiovascular complications [14-15]. Statistical figures of USA and Western Europe showed that chronic wounds affect 1 to $2 \%$ of the population that in turn cost around 1 to $3 \%$ expenditure incurred in the annual healthcare budgets [16-18]. During the year 2012 and 2013, the census done by UK National Health Service (NHS) revealed that 2.2 million adult patients ( $4.5 \%$ of the total population) were treated for chronic wounds that cost 0 ? 4.5 to 95.0 billion per annum [14]. Out of these subjects, over 1 million patients were suffering from diabetes mellitus, venous and arterial insufficiency [14]. Furthermore, another study done on Welsh population, it was shown that chronic wounds affected $6 \%$ (190 thousand individuals) population in the UK that incurred around 5.5\% budget in Wales (or over 2300 million per annum) [17]. To generate the reliable figures evidences based systemic review PROSPERO and CRD42016037496 has released their figures on the burden of chronic wounds [19].

\section{Classification of Surgical Wounds}

The American College of Surgeons wound classification schema adopted surgical wound classification [20]. This system implicated for postoperative grading depending upon the extent of microbial load or infection that predict the occurrence of the infection at the surgical site in patients. The college classified the wound into four major classes as described below:

a) Class I/Clean Wounds: This is an uninfected surgical wound devoid of any inflammation. Moreover, clean wounds are primarily closed. It is to believe that surgical incisions that are created post non-penetrating trauma might be included in this type of wound class if they meet the criteria.

b) Class II/Clean-Contaminated Wounds: This a type of surgical wound in which there is entry of alimentary, respiratory or genital tracts under controlled conditions devoid of unusual contamination. This class includes surgical procedures done with vagina, oropharynx, biliary tract and appendix without any sign of infection at the site.

c) Class III/Contaminated Wounds: These wounds are open, fresh and occurred due to accidents. Moreover, surgical procedures involving major break of sterile site (e.g. open cardiac procedure) comes under this class. These are characterized by acute, nonpurulent inflammation.

d) Class IV/Infected or Dirty Wounds: These are old traumatic wounds with sustained devitalized tissue and concluded into chronic infection with severe inflammation with perforated viscera. 


\section{Concept of Vasculogenesis and Angiogenesis}

Vasculogenesis clearly state the formation of new vessels from the progenitor cells that together form primitive vascular networks. This mechanism which prominently takes place during development showed contrast behavior with angiogenesis, which denotes new vascular formation from pre-established vasculature [21]. Angiogenesis predominantly occurs during the embryonic and post-natal development which shows quite rare occurrence in healthy adult phase except in the female reproductive setup that includes ovary, endometrial lining of the uterus and placenta [22-23]. Pro-angiogenic and anti-angiogenic factors are controlling the mechanism of physiological and pathological angiogenesis process. The non-pathologic angiogenesis is characterized by vessels formation by their virtue of refinement, integrity and ability to transmit nutrient and essential factors for the tissues in a controlled environment [24]. Solid tumor growth and fibrosis is characterized by disarrayed and runaway angiogenesis [24]. In healthy state, the homeostatic expression of pro-angiogenic factors and their corresponding receptors seems to be in maintained manner, but in the diseases or any pathologic state the expression of these factors shows up regulation that includes expression of proangiogenic vascular epithelial growth factors (VEGFs), fibroblast growth factors (FGFs). Their unregulated expression leads to unfettered formation of vessel beds and proliferation along with endothelial cells migration [25]. Pathological angiogenesis is occasionally associated with malignancy due to excessive and uncontrolled angiogenesis that may lead to the development of the tumor like entities [26]. Uncontrolled angiogenesis may also contribute in pathophysiology of diabetes associated comorbidities that may become fatal to diabetic patients [27].

\section{Wound Angiogenesis in Healthy State}

Wound healing is a complicated mechanism that comprises of series of stages including hemostasis, inflammation, proliferation and remodeling of tissue [28]. In healthy state devoid of any injury, the vasculature is in the phase of dormancy, in which the blood vasculatures are sufficiency perfused for nutrients and oxygen supplement to the tissues. The adequate expression of pro and anti-angiogenic factors enabled proper functional vasculature, and during the state of injury, this homeostasis impaired, leading to hypoxia state [28]. During the state of hypoxia, the innate immune system harbors leuckocytes to the injury site along with neutrophils leading to the acute phase of inflammation [29]. Influx of neutrophils initiate macrophages recruitment at the injury site that compels the tissue to undergo in proliferative phase in wound healing. Macrophages, new capillaries emergence and relaxed connective tissue represented by edema form tissue granulation [30]. Moreover due to oxygen gradient developed at the wound site, several proangiogenic factors showed their presence. Most notable among them is vascular epidermal growth factor (VEGF) that produces downstream of hypoxia in wounds.
Hypoxia activates the expression of transcription factor namely hypoxia-inducible factor-1 (HIF-1) that promote the mechanism of angiogenesis by upregulation of VEGF-A that covalently binds to endothelial receptors and induces signal for vasculature formation at wound site [31]. Moreover, wound angiogenesis is characterized by generation of dishonored and inadequate vasculature followed by deformed capillaries bed with unguided sprouts and torturous network [24]. The vasculature proliferation is presented as muddled, vigorous and plenty is also contrasted by maturation, a mechanism that refines and chooses for competent nascent vasculature to become stable and reliable vessels with integrity in function similar to pre-injured state [32]. Smooth muscle cell recruitment also termed as pericytes recruited post capillary refinement process [32]. These pericytes are equally important for the maturation and stabilization of newly formed vasculature at wound site. Pericytes recruited capillaries shows neutrality against antiangiogenic factors which expresses during remodeling phase of the wound repair. Moreover, wound angiogenesis, enhances active ingredient production at the site that includes pigment epithelium derived factor (PEDF), a member of serine protease inhibitor family and sprout-2 (SPRY2) [33]. These two factors show powerful haemostatic behavior at wound site. Additionally, to these factors another set of factors including angiopoietins 1 (Ang 1) and 2 (Ang 2) also shows expression at wound site to resume normal progression of wound capillaries and nourish their growth. Post completion of remodeling phase of wound angiogenesis and healing, the tissue regain its integrity and normal vasculature with proper permeability, blood flow [34]. Oxygen demand of early wound healing stages was return to normoxia that activate signals for immune mediators and several pro-angiogenic factors to return to normal level (preinjury levels) [35].

\section{Wound Angiogenesis in Diabetes Mellitus}

Angiogenesis of wound in healthy state, as describe in the above section is a homeostatic balance between new vasculature formation and cell proliferation upto cell maturation. The diabetes mellitus adversely affect this balance and perturb proper wound healing, cell proliferation, tissue regeneration and creation of healthy and sound vasculature. Impairment in the vasculature integrity is also a prime characteristic of diabetes mellitus. Sustained hyperglycemia during diabetes mellitus has been reported for progression of vascular complications in animal and clinical studies [36]. The uncontrolled glycemic control is the prime cause of micro-and macro vascular complications including diabetic retinopathy, nephropathy, neuropathy and cardio vascular disease. These complications ultimately lead to impairment in wound healing and badly affect angiogenesis mechanism. Endothelial cells (ECs) counteract with sustained and prolonged hyperglycemia lead to integrity and functional loss that in turn increased susceptibility for apoptosis along with many other impairments [37]. In diabetes, a study showed that presence of circulating free ECs 
in blood is the marker of coronary heart disease [38,39]. It is interesting to know that diabetic wound is devoid of angiogenic activities that may decline wound angiogenesis and initiate several pathological mechanisms. In diabetic retinopathy (DR) pathological complications like microaneurysms, hemorrhages and vascular edema is the resultant of excessive angiogenesis [40]. DR is also characterized by loss of pericytes cells from the retinal capillary that contribute to vascular edema and leakage that in turn results in establishment of hypoxia. Hypoxia, due to edema may activate HIF-1 expression in an abrupt way, that upregulate the expression of VEGF-A and results in increased vascular permeability. Similarly diabetic nephropathy (DN) is also characterized by impaired wound angiogenesis that may lead to damage in glomerular filtration setup. In previously published study, it is evident that in the initial stages of DN, excess VEGF-A releases that increases the permeability of vessels and enhances the ECs proliferation and inhibit apoptosis [41].

In addition to DR and DN, delayed wound healing is also a characteristic of diabetes associated complication. In diabetes mellitus, wounds render improper angiogenesis and showed impart vascularity and density of ECs in capillary [41]. Wound healing in diabetes is of great concern as it got delayed. Macrophages are interesting candidate for wound healing and repair that have been shown with impaired functions in state of uncontrolled hyperglycemia [41]. During healthy state wound repair, macrophages enable switching of proinflammatory to pro-reparative phenotype that support tissue repair. Moreover, in diabetic state, these macrophages are deficit with this function and hence the tissue repair process got hamper. One of the known animal model quoted in the literature is $\mathrm{db} / \mathrm{db}$ mouse that is genetic model for diabetes, obesity and dyslipidemia and the studies shown for delayed wound healing and impaired angiogenesis on this model [42].

\section{Conclusion}

Wound healing is a complex biological mechanism that follows hemostasis, inflammation, cell proliferation and tissue remodeling leading to proper vasculature with normal function and identity. Varied cells are involved in the wound angiogenesis that includes macrophages, endothelial cells along with keratinocytes. The burden of chronic wounds leads to financial deficit to any country that in turn imposes extra burden to the society. The healthy state wound angiogenesis follows classical pathways for repair and healing, but in diabetic wounds these classical pathways got hampered that delayed the healing and may lead to co-morbidities associated with diabetes mellitus.

\section{References}

1. (2009) Diagnosis and Classification of Diabetes Mellitus. Diabetes Care 32(1): 62-67.

2. Pengzi Zhang, Nanjing, China, Jing Lu, Yali Jing, et al. (2017) Global epidemiology of diabetic foot ulceration: a systematic review and meta-analysis. Journal Annals of Medicine 49(2): 06-116.
3. Bickers DR, Lim HW, Margolis D, Goodman C (2006) The burden of skin diseases: 2004 a joint project of the American Academy of Dermatology Association and the Society for Investigative Dermatology. J Am Acad Dermato 55(3): 490-500.

4. Joret MO, Dean A, Cao C Stewart J, Bhamidipaty V (2016) The financial burden of surgical and endovascular treatment of diabetic foot wounds. J Vasc Surg 64(3): 648-655.

5. Guest JF, Ayoub N, Mcllwraith, Ijeoma UchegbuT, Alyson Gerrish, et al. (2015) Health economic burden that wounds impose on the National Health Service in the UK. BMJ 5: 009283.

6. Schultz GS (1999) Molecular Regulation of Wound Healing. In: Acute and Chronic Wounds. Nursing Management Bryant RA ( $2^{\text {nd }}$ Edn.), WB Saunders Publishers, USA.

7. Menke NB, KR Ward, TM Witten, DG Bonchev, RF Diegelmann (2007) Impaired wound healing. Clin. Dermatol 25(1): 19-25.

8. Monteiro Soares M, Boyko EJ, Ribeiro J, Ribeiro I, Dinis Ribeiro M (2012) Predictive factors for diabetic foot ulceration: a systematic review. Diabetes Metab Res Rev 28(7): 574-600.

9. GS Lazarus, DM Cooper, DR Knighton, RE Percoraro, G Rodeheaver, et al. (1994) Definitions and guidelines for assessment of wounds and evaluation of healing. Wound Repair Regen 2(3): 165-170.

10.F Werdin, M Tennenhaus, HE Schaller, HO Rennekampff (2009) Evidence-based management strategies for treatment of chronic wounds. Eplasty 9: 19.

11. K Järbrink, G Ni, H Sönnergren, A Schmidtchen, C Pang, et al. (2016) Prevalence and incidence of chronic wounds and related complications: a protocol for a systematic review. Syst Rev 5(1): 152.

12. CK Sen, GM Gordillo, S Roy, R Kirsner, L Lambert, et al. (2009) Human skin wounds: a major and snowballing threat to public health and the economy. Wound Repair Regen 17(6): 763-771.

13. C Wicke, A Bachinger, S Coerper, S Beckert, MB Witte, et al. (2009) Aging in- fluences wound healing in patients with chronic lower extremity wounds treated in a specialized wound care center. Wound Repair Regen 17(1): 25-33.

14. EW Gregg, JE Shaw (2017) Global health effects of overweight and obesity. N Engl J Med 377: 80-81.

15. The GBD 2015 Obesity Collaborators, health effects of overweight and obesity in 195 countries over 25 years, N Engl J Med 37: 13-27.

16. RG Frykberg, J Banks, (2015) Challenges in the treatment of chronic wounds. Adv Wound Care New York 4(9): 560-582.

17.JF Guest, N Ayoub, T Mcllwraith, I Uchegbu, A Gerrish, et al. (2017) Health economic burden that different wound types impose on the UK's national health service. Int Wound J 14(2): 322-330.

18. CJ Phillips, I Humphreys, J Fletcher, K Harding, G Chamberlain, et al. (2016) Estimating the costs associated with the management of patients with chronic wounds using linked routine data. Int Wound J 13(6): 1193-1197

19. K Järbrink, G Ni H, Sönnergren A, Schmidtchen C, Pang R, et al. (2017) The humanistic and economic burden of chronic wounds: a protocol for a systematic review. Syst Rev 6: 15.

20. Berard F, Gandon J (1964) Postoperative wound infections: the influence of ultraviolet irradiation of the operating room and of various other factors. Ann Surg 160(2): 1-192.

21. Kolte D, McClung JA, Aronow WS (2015) Vasculogenesis and Angiogenesis. In Translational Research in Coronary Artery Disease: Pathophysiology to Treatment. Cambridge, MA, USA, pp. 49-65. 
22. Geudens I, Gerhardt H (2011) Coordinating cell behaviour during blood vessel formation. Development 138(21): 4569-4583.

23. Reynolds LP, Grazul Bilska AT, Redmer DA (2002) Angiogenesis in the female reproductive organs: Pathological implications. Int J Exp Pathol 83(4): 151-163.

24. Fukumura DAI, Jain RK (2008) Imaging angiogenesis and the microenvironment. APMIS 116(8): 695-715.

25. Papetti, M, Herman IM (2002) Mechanisms of normal and tumorderived angiogenesis. AJP Cell Physiol 282(5): 947-970.

26. Kerbel RS (2008) Tumor angiogenesis. N Engl J Med 358: 2039-2049.

27. Fowler MJ (2011) Microvascular and macrovascular complications of diabetes. Clin Diabetes 29: 116-122.

28. Guo S, DiPietro LA (2010) Factors affecting wound healing. J Dent Res 89(3): 219-229.

29. Turabelidze A, Dipietro LA (2013) Inflammation andWound Healing. In Oral Wound Healing: Cell Biology and Clinical Management, pp. 3956.

30. Koh TJ, DiPietro LA (2011) Inflammation and wound healing: The role of the macrophage. Expert Rev Mol Med 13: 23.

31. Liu L, Marti GP, Wei X, Zhang X, Zhang H, et al. (2008) Age-dependent Impairment of HIF-1 expression in diabetic mice: Correction with electroporation-facilitated gene therapy increases wound healing, angiogenesis, and circulating angiogenic cells. J Cell Physiol 217(2): 319-327.

32. Chen RR, Silva EA, Yuen WW, Mooney DJ (2007) Spatio-temporal VEGF and PDGF delivery patterns blood vessel formation and maturation. Pharm Res 24(2): 258-264.
33. Pries AR, Secomb TW (2014) Making Microvascular Networks Work: Angiogenesis, Remodeling, and Pruning. Physiology 29(6): 446-455.

34. Carmeliet P (2005) Angiogenesis in life, disease and medicine. Nature 438(7070): 932-936.

35. Hickey MM, Simon MC (2006) Regulation of Angiogenesis by hypoxia and hypoxia-inducible factors. Curr Top Dev Biol 76: 217-257.

36. Altabas V (2015) Diabetes, endothelial dysfunction, and vascular repair: What should a diabetologist keep his eye on? Int J Endocrinol p. 1-14.

37. Yu JQ, Liu XF, Chin LK, Liu AQ, Luo KQ (2013) Study of endothelial cell apoptosis using fluorescence resonance energy transfer (FRET) biosensor cell line with hemodynamic microfluidic chip system. Lab Chip 13(14): 2693-2700.

38. McClung JA, Naseer N, Saleem M, Rossi GP, Weiss MB, et al. (2005) Circulating endothelial cells are elevated in patients with type 2 diabetes mellitus independently of HbA1c. Diabetologia 48(2) 345350 .

39. Kota Sk, Meher L, Jammula S, Kota S, Krishna SVS, et al. (2012) Aberrant angiogenesis: The gateway to diabetic complications. Indian J Endocrinol Metab 16(6): 918-930

40. McGinn S, Saad S, Poronnik P, Pollock CA (2003) High glucose-mediated effects on endothelial cell proliferation occur via p38 MAP kinase. Am J Physiol Endocrinol Metab 285(4): 708-717.

41. Dinh T, Veves A (2005) Microcirculation of the Diabetic Foot. Curr Pharm Des 11(18): 2301-2309.

42. Michaels J, Churgin SS, Blechman KM, Greives MR, Aarabi S, et al. (2007) $\mathrm{db} / \mathrm{db}$ mice exhibit severe wound-healing impairments compared with other murine diabetic strains in a silicone-splinted excisional wound model. Wound Repair Regen 15(5): 665-670.

\section{Your next submission with Juniper Publishers will reach you the below assets}

- Quality Editorial service

- Swift Peer Review

- Reprints availability

- E-prints Service

- Manuscript Podcast for convenient understanding

- Global attainment for your research

- Manuscript accessibility in different formats

( Pdf, E-pub, Full Text, Audio)

- Unceasing customer service

Track the below URL for one-step submission

https://juniperpublishers.com/online-submission.php 